\title{
Model to Enhance Site-Specific Estimation of Leaf Wetness Duration
}

\author{
K. S. Kim, Department of Agronomy; S. E. Taylor, Department of Agronomy; M. L. Gleason, Department of Plant \\ Pathology; and K. J. Koehler, Department of Statistics, Iowa State University, Ames 50011
}

\begin{abstract}
Kim, K. S., Taylor, S. E., Gleason, M. L., and Koehler, K. J. 2002. Model to enhance site-specific estimation of leaf wetness duration. Plant Dis. 86:179-185.

The ability of empirical models to enhance accuracy of site-specific estimates of leaf wetness duration (LWD) was assessed for 15 sites in Iowa, Nebraska, and Illinois during May to September of 1997, 1998, and 1999. Enhanced estimation of LWD was obtained by applying a 0.3$\mathrm{m}$ height correction to SkyBit wind-speed estimates for input to the classification and regression tree/stepwise linear discriminant (CART/SLD) model (CART/SLD/Wind model), compared to either a proprietary model (SkyBit wetness) or to the CART/SLD model using wind speed estimates for a 10-m height. The CART/SLD/Wind model estimated LWD more accurately than the other models during dew-eligible (20:00 to 9:00) as well as dew-ineligible (10:00 to 19:00) periods, and for the period 20:00 to 9:00 regardless of rain events. Improvement of LWD estimation accuracy was ascribed to both the hierarchical structure of decision-making in the CART procedure and wind speed correction. Accuracy of the CART/SLD/Wind model identifying hours as wet or dry varied little among the 15 sites, suggesting that this model may be desirable for estimating LWD from site-specific data throughout the midwestern United States.
\end{abstract}

Additional keywords: disease warning system, integrated pest management, weather models
One of the most important factors influencing the outbreak and severity of foliar diseases is the duration of leaf wetness from dew deposition, rainfall, fog, or irrigation $(7,17)$. Both on-site measurements and wetness estimation models have been used to assess leaf wetness duration (LWD). Using sensors and data-loggers to obtain weather measurements is unattractive to many growers because this hardware is costly and requires significant effort to install, maintain, and download. Models may provide alternatives for determining LWD without the difficulty of measuring it. Various LWD models have been developed and refined to aid imple-

Corresponding authors: K. S. Kim and M. L. Gleason

E-mail: makd@iastate.edu

and mgleason@iastate.edu

Journal Paper No. J-19438 of the Iowa Agriculture and Home Economics Experiment Station, Ames, Act and State of Iowa Funds.

Funding was provided by grants from the North Central Region IPM Competitive Grants Program of the USDA and the Region VII Pesticide Environmental Stewardship Program of the EPA.

Accepted for publication 12 October 2001.

Publication no. D-2001-1217-02S

(C) 2002 The American Phytopathological Society Iowa. Project No. 3564, and supported by Hatch mentation of crop disease-warning systems $(2,8,14)$.

LWD estimation models are classified into empirical and physical types. The latter utilize energy balance equations (8), whereas the former generally require fewer input variables and depend on statistical methods. A relative humidity $(\mathrm{RH})$ model that assumes occurrence of leaf wetness whenever RH $>90 \%$ (21) is an example of a simple empirical model. Gleason et al. (6) developed a nonparametric empirical model for estimating dew duration using classification and regression tree (CART) analysis with stepwise linear discriminant analysis (SLD). Estimation of LWD by the CART/SLD model was more accurate than by the $\mathrm{RH}>90 \%$ model or an artificial neural network model $(2,6)$.

A potential advantage of models is that they may be used to estimate LWD from either on-site weather measurements, offsite or remotely estimated data, or both. Modeling efforts to estimate LWD from off-site weather inputs have been reported. For example, SkyBit, Inc. (Bellefonte, PA) provides model-derived LWD estimates from estimated input parameters. In separate studies, mean underestimation of LWD by SkyBit was $3.4 \mathrm{~h} /$ day (5) and 1.4 h/day (20). Magarey (13) reported that his water budget model failed to accurately predict LWD with off-site, computer-estimated data because the model was sensitive to errors in input variables, especially RH. Energy balance models consider all input parameters simultaneously; therefore, their accuracy may be constrained by inaccuracy in one or more of the inputs. Models that are more robust to errors in input variables may be able to estimate LWD with a higher degree of accuracy. Corrected wind speed estimates from a standard meteorological datum (e.g., $10 \mathrm{~m}$ ) to crop canopy level also could enhance LWD model accuracy because wind speed is usually slower near the surface than aloft.

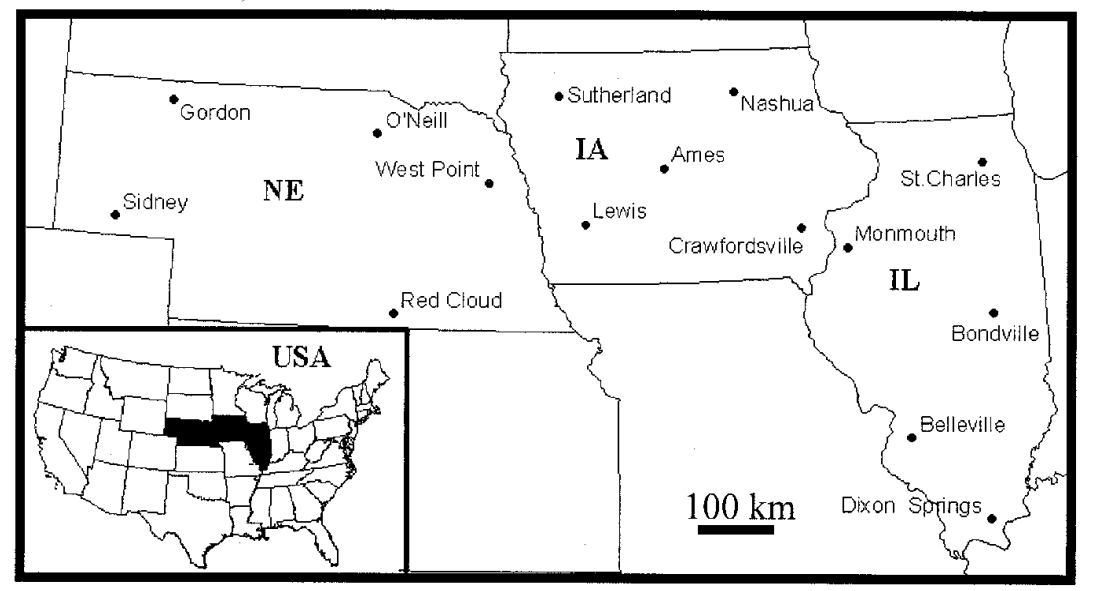

Fig. 1. Locations of weather stations where leaf wetness duration, air temperature, wind speed, and relative humidity were measured and for which SkyBit, Inc. weather data were estimated during May to September of 1997, 1989, and 1999. 
The objectives of this study were to (i) estimate LWD with the CART/SLD model, using site-specific weather data with corrected wind speed, and (ii) compare the accuracy of estimates to those obtained by a proprietary, off-site, mesoscale model (SkyBit, Inc).

\section{MATERIALS AND METHODS}

Wetness measurement. Model-derived estimates of hourly weather data, provided by SkyBit, Inc. for 15 weather stations in Iowa, Illinois, and Nebraska (Fig. 1), were received daily by electronic mail from May to September in 1997, 1998, and 1999. SkyBit data were estimates of RH, air temperature, wind speed, and leaf wetness duration $(0=$ dry, $1=$ wet $)$ for each site based on interpolation of measurements at regional weather stations (5).

LWD, air temperature, RH, and rainfall amount were obtained during the study period at the same sites for which the SkyBit data were received. Flat, printed-circuit, electronic wetness sensors (Model 237; Campbell Scientific, Logan, UT) were deployed at a $45^{\circ}$ angle, facing north, at level, unobstructed sites on managed turfgrass, $0.3 \mathrm{~m}$ above the ground. Sensor surfaces were painted with latex paint of proprietary composition (R. Olson, Savannah, GA, personal communication) in order to increase sensitivity to small water droplets and to approximate the emissivity of plant leaves $(1,3,12)$. These sensors were determined in extensive field tests to be accurate within $1 \mathrm{~h}$ /day $(12,15)$. When wetness sensors detected wetness for $\geq 30$ min in an hour, the hour was scored as wet ("1"); when wetting occurred for <30 min, the hour was scored as dry ("0").

Wetness estimation models. A proprietary model whose output was provided by SkyBit, Inc. (designated the SkyBit Wetness model) and the CART/SLD model, using SkyBit weather data (6) as inputs, were evaluated (Table 1). The CART/SLD model utilizes dew point depression (air temperature - dew point temperature; DPD), wind speed, and RH as variables. Dew point temperature was calculated from air temperature data and $\mathrm{RH}$ using the Clausius-Clapeyron equation (18). The CART/SLD model assigns hourly data to one of four categories according to threshold values of $3.7^{\circ} \mathrm{C}$ for DPD, $2.5 \mathrm{~m} / \mathrm{s}$ for wind speed (Wind), and $87.8 \%$ for $\mathrm{RH}$ (Fig. 2). Hours are classified as dry if either DPD $\geq 3.7^{\circ} \mathrm{C}$ (category 1 ) or $\mathrm{RH}<$ $87.8 \%$ and Wind $\geq 2.5 \mathrm{~m} / \mathrm{s}$ (category 4 ). Hours in categories 2 and 3 are classified as either dry or wet by subsequent SLD analysis. For category 2, in which DPD < $3.7^{\circ} \mathrm{C}$ and Wind $<2.5 \mathrm{~m} / \mathrm{s}$, hours are classified as wet if the following inequality is met:

$\left(1.6064 \sqrt{T_{\text {air }}}+0.0036 T_{\text {air }}^{2}+0.1531 \mathrm{RH}\right.$

-0.4599 Wind $\times$ DPD $\left.-0.0035 T_{\text {air }} \times \mathrm{RH}\right)>14.4674$

(Inequality 1).

For category 3 , where DPD $<3.7^{\circ} \mathrm{C}$, Wind $\geq$ $2.5 \mathrm{~m} / \mathrm{s}$, and $\mathrm{RH} \geq 87.8 \%$, hours are classified as wet if the following inequality is met:

$\left(0.7921 \sqrt{T_{\text {air }}}+0.0046 \mathrm{RH}-2.3889\right.$ Wind $-0.0390 T_{\text {air }}$ $\times$ Wind +1.0613 Wind $\times$ DPD) $>37.0$

(Inequality 2).

where $T_{\text {air }}$ is air temperature $\left({ }^{\circ} \mathrm{C}\right), \mathrm{RH}$ is relative humidity ( 0 to $100 \%$ ), DPD is dew point depression $\left({ }^{\circ} \mathrm{C}\right)$, and Wind is wind speed $(\mathrm{m} / \mathrm{s})(6)$.
Wind speed correction. Wind speed near the earth's surface differs from wind speed aloft because the surface exerts a drag force on air flow. A crop canopy can act as a major component of the drag force. In the present study, a $0.3-\mathrm{m}$ height was chosen to approximate the top of the canopy of low-growing crops such as strawberries or melons.

SkyBit estimates of wind speed at 10$\mathrm{m}$ height were corrected to canopy height $(0.3 \mathrm{~m})$ using the logarithmic wind profile equation given by Rosenberg et al. (16):

$\frac{u_{1}}{u_{2}}=\frac{\ln \left(z_{1}-d\right)-\ln z_{0}}{\ln \left(z_{2}-d\right)-\ln z_{0}}$

(Equation 1)

where $u_{1}$ and $u_{2}$ are the mean wind speed at the height of $z_{1}$ and $z_{2}$, respectively; $d$ is zero plane displacement; and $z_{0}$ is rough-

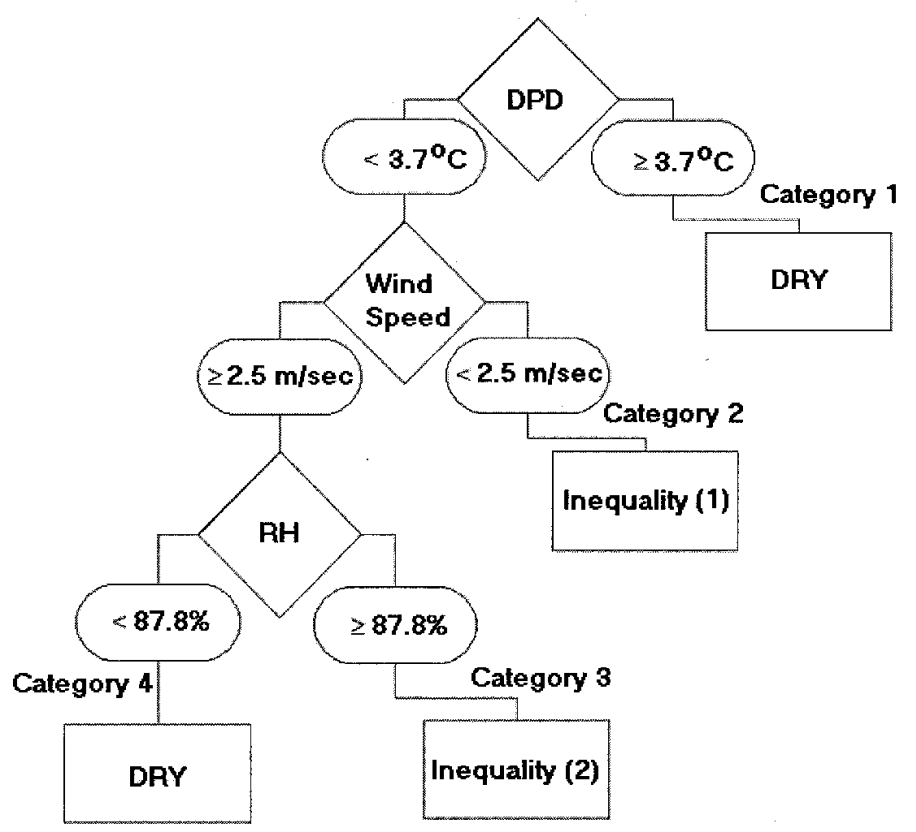

$\left(1.6064 \sqrt{T_{\text {air }}}+0.0036 T_{\text {air }}^{2}+0.1531 R H-0.4599\right.$ Wind $\left.\times D P D-0.0035 T_{\text {air }} \times R H\right)>14.4674$

(Inequality 1).

$\left(0.7921 \sqrt{T_{\text {air }}}+0.0046 R H-2.3889\right.$ Wind $-0.0390 T_{\text {air }} \times$ Wind +1.0613 Wind $\left.\times D P D\right)>37.0$

(Inequality 2).

Fig. 2. Classification tree for prediction of wetness duration (6). DPD = dew point depression, which is the difference between the air temperature and the dew point. DRY indicates that hours are categorized as dry $(0)$.

Table 1. Summary of leaf wetness duration-estimation models evaluated in this study

\begin{tabular}{lcll}
\hline Model name & Input data source & \multicolumn{1}{c}{ Base model } & \multicolumn{1}{c}{ Wind speed input } \\
\hline SkyBit Wetness & SkyBit & Proprietary (SkyBit, Inc.) & Wind speed estimated at 1-m height \\
CART/SLD/SkyBit ${ }^{\mathrm{a}}$ & SkyBit & CART/SLD & Wind speed estimated at 10-m height (from SkyBit). \\
CART/SLD/Wind & SkyBit & CART/SLD & Wind speed estimate of SkyBit corrected to 0.3-m height. \\
\hline
\end{tabular}

${ }^{\mathrm{a}} \mathrm{CART} / \mathrm{SLD}=$ classification and regression tree/stepwise linear discriminant (6). 
ness length. In this study, $z_{1}$ was the reference level $(10 \mathrm{~m})$ and $z_{2}$ was the height $(0.3 \mathrm{~m})$ of the wetness sensors. In order to apply the logarithmic wind profile equation, it was assumed that neutral atmospheric stability existed during all days and that adequate fetch, or upwind distance, of uniform roughness over the surface was available (16).

Analysis of wetness estimation. Measurements from wetness sensors and deviations of model simulations from those values were assumed to represent true wetness occurrence and errors, respectively. Days that had any apparent measurement errors (e.g., out-of-range values such as $>100 \% \mathrm{RH}$ or missing data), which comprised $6.6 \%$ of the total number of days during the study period, were excluded from the analysis. Daily data sets began at 12:00 and ended at 11:00, respec- tively, in order to include entire dew periods within data records.

Mean error (ME) was calculated by averaging differences between measured and model-estimated LWD for 24-h periods. Mean absolute error (MAE) was also computed by totaling hours in which measurement and estimation disagreed during 24-h periods and averaging the totals over the study period. ME provided a measure of the tendency to over- or underestimate LWD, whereas MAE assessed overall accuracy.

Dew can form after sunset and dry off within several hours after sunrise; therefore, 24-h periods were partitioned into dew-eligible (from 20:00 to 9:00) and dewineligible periods (from 10:00 to 19:00). Nights were grouped into those with and without measured rainfall $(\geq 0.25$ $\mathrm{mm} / \mathrm{night}$ ), and analysis of variance was used to determine whether model performance was affected by occurrence of rainfall.

Sensitivity analysis was performed in order to examine the response of each model to changes in $\mathrm{RH}$ and Wind. Only hours with $\mathrm{RH} \geq 50 \%$ were used in the analysis because wetness rarely was measured at $\mathrm{RH}<50 \%$. Wind speed was partitioned into low $(<1.3 \mathrm{~m} / \mathrm{s})$, middle $(\geq 1.3 \mathrm{~m} / \mathrm{s}$ and $\leq 3.6 \mathrm{~m} / \mathrm{s})$, and high $(>3.6$ $\mathrm{m} / \mathrm{s}$ ) ranges, corresponding to the twentyfifth and seventy-fifth percentiles of the hourly wind speed estimates. Correlation between SkyBit errors and accuracy of models, defined as the proportion of hours in which each model correctly estimated occurrence or absence of wetness, was analyzed to examine whether these errors significantly affect model performance.

Table 2. Mean error (ME) and mean absolute error (MAE) for estimation of wetness duration (h/day)

\begin{tabular}{|c|c|c|c|c|c|c|c|}
\hline \multirow[b]{2}{*}{ City } & \multirow[b]{2}{*}{$n^{\mathrm{c}}$} & \multicolumn{3}{|c|}{ ME (h/day) (SEM)a } & \multicolumn{3}{|c|}{$\mathbf{M A E}^{\mathbf{b}}$} \\
\hline & & SkyBit Wetness & CART/SLD/SkyBitd & CART/SLD/Wind & SkyBit Wetness & CART/SLD/SkyBit & CART/SLD/Wind \\
\hline Ames, IA & 358 & $-1.3(0.35)$ & $-4.8(0.24)$ & $0.6(0.24)$ & 7.7 & 6.0 & 4.6 \\
\hline Lewis, IA & 380 & $1.7(0.28)$ & $-2.6(0.21)$ & $2.6(0.19)$ & 6.7 & 4.5 & 4.7 \\
\hline Nashua, IA & 360 & $0.4(0.32)$ & $-3.4(0.23)$ & $2.4(0.20)$ & 6.8 & 4.9 & 4.2 \\
\hline Sutherland, IA & 380 & $-1.7(0.33)$ & $-5.5(0.23)$ & $0.4(0.23)$ & 7.2 & 6.0 & 4.3 \\
\hline Crawfordsville, IA & 365 & $-1.8(0.37)$ & $-4.7(0.24)$ & $0.9(0.23)$ & 7.8 & 5.6 & 4.2 \\
\hline Belleville, IL & 267 & $-1.7(0.48)$ & $-2.6(0.25)$ & $1.3(0.29)$ & 8.1 & 4.3 & 4.1 \\
\hline Bondville, IL & 327 & $-2.5(0.42)$ & $-5.7(0.24)$ & $0.0(0.28)$ & 8.6 & 6.3 & 4.3 \\
\hline Dixon Springs, IL & 325 & $-1.9(0.32)$ & $-2.8(0.22)$ & $1.1(0.18)$ & 7.3 & 4.1 & 3.2 \\
\hline Monmouth, IL & 322 & $-1.4(0.41)$ & $-4.5(0.28)$ & $0.6(0.28)$ & 7.6 & 5.4 & 4.7 \\
\hline St. Charles, IL & 319 & $-2.1(0.40)$ & $-5.3(0.22)$ & $-0.6(0.26)$ & 7.5 & 5.8 & 4.1 \\
\hline Red Cloud, NE & 388 & $-2.0(0.33)$ & $-5.9(0.22)$ & $-1.0(0.23)$ & 7.0 & 6.4 & 4.4 \\
\hline Gordon, NE & 386 & $-2.3(0.34)$ & $-6.8(0.24)$ & $-3.2(0.24)$ & 7.0 & 7.1 & 5.0 \\
\hline O'Neill, NE & 318 & $-2.0(0.38)$ & $-5.4(0.26)$ & $-1.0(0.24)$ & 7.0 & 6.2 & 4.7 \\
\hline Sidney, NE & 384 & $0.2(0.26)$ & $-4.7(0.24)$ & $-0.6(0.18)$ & 4.9 & 4.9 & 3.3 \\
\hline West Point, NE & 247 & $-3.0(0.36)$ & $-6.5(0.27)$ & $-1.7(0.20)$ & 7.8 & 6.7 & 3.5 \\
\hline All 15 locations & 5,126 & $-1.4(0.09)$ & $-4.8(0.06)$ & $0.1(0.06)$ & 7.2 & 5.6 & 4.2 \\
\hline
\end{tabular}

a $\mathrm{ME}=$ mean error $(\Sigma($ estimated - measured $) / \mathrm{n})$ and $\mathrm{SEM}=$ standard error of the mean difference.

b MAE = mean absolute error $(\Sigma \mid$ estimated-measuredl $/ \mathrm{n})$.

c CART/SLD = classification and regression tree/stepwise linear discriminant.

$\mathrm{d}$ Number of 24-h periods included in the analysis.

Table 3. Mean error (ME) of model-estimated wetness duration (h/period) for the periods 10:00 to 19:00 and 20:00 to 9:00

\begin{tabular}{|c|c|c|c|c|c|c|c|}
\hline \multirow[b]{2}{*}{ City } & \multirow[b]{2}{*}{$n^{\mathbf{b}}$} & \multicolumn{3}{|c|}{ ME (h/period, 10:00 to 19:00) (SEM)a } & \multicolumn{3}{|c|}{ ME (h/period, 20:00 to 9:00) (SEM) } \\
\hline & & $\begin{array}{c}\text { SkyBit Wet- } \\
\text { ness }\end{array}$ & $\begin{array}{c}\text { CART/SLD/ } \\
\text { SkyBitc }^{c}\end{array}$ & $\begin{array}{c}\text { CART/SLD/ } \\
\text { Wind }\end{array}$ & $\begin{array}{c}\text { SkyBit } \\
\text { Wetness }\end{array}$ & $\begin{array}{c}\text { CART/SLD/ } \\
\text { SkyBit }\end{array}$ & $\begin{array}{c}\text { CART/SLD/ } \\
\text { Wind }\end{array}$ \\
\hline Ames, IA & 358 & $1.6(0.12)$ & $-0.5(0.06)$ & $0.3(0.07)$ & $-2.9(0.28)$ & $-4.3(0.23)$ & $0.4(0.21)$ \\
\hline Lewis, IA & 380 & $1.9(0.12)$ & $-0.5(0.07)$ & $0.4(0.08)$ & $-0.3(0.23)$ & $-2.1(0.18)$ & $2.3(0.17)$ \\
\hline Nashua, IA & 360 & $1.8(0.12)$ & $-0.5(0.08)$ & $0.5(0.09)$ & $-1.4(0.24)$ & $-2.9(0.20)$ & $1.8(0.16)$ \\
\hline Sutherland, IA & 380 & $1.2(0.11)$ & $-0.6(0.07)$ & $0.0(0.07)$ & $-3.0(0.27)$ & $-4.9(0.21)$ & $0.3(0.20)$ \\
\hline Crawfordsville, IA & 365 & $1.6(0.12)$ & $-0.5(0.07)$ & $0.4(0.08)$ & $-3.4(0.29)$ & $-4.2(0.21)$ & $0.5(0.20)$ \\
\hline Belleville, IL & 267 & $1.6(0.16)$ & $-0.3(0.05)$ & $0.3(0.08)$ & $-3.2(0.36)$ & $-2.3(0.24)$ & $1.0(0.26)$ \\
\hline Bondville, IL & 327 & $1.5(0.15)$ & $-0.6(0.07)$ & $0.3(0.10)$ & $-4.0(0.32)$ & $-5.1(0.23)$ & $-0.3(0.23)$ \\
\hline Dixon Springs, IL & 325 & $1.4(0.12)$ & $-0.4(0.07)$ & $0.3(0.07)$ & $-3.3(0.24)$ & $-2.4(0.20)$ & $0.9(0.16)$ \\
\hline Monmouth, IL & 322 & $1.3(0.14)$ & $-0.6(0.08)$ & $0.1(0.08)$ & $-2.7(0.33)$ & $-3.9(0.25)$ & $0.5(0.25)$ \\
\hline St. Charles, IL & 319 & $1.2(0.12)$ & $-0.5(0.07)$ & $0.1(0.07)$ & $-3.3(0.32)$ & $-4.7(0.20)$ & $-0.7(0.23)$ \\
\hline Red Cloud, NE & 388 & $1.1(0.10)$ & $-0.4(0.06)$ & $-0.0(0.06)$ & $-3.1(0.27)$ & $-5.5(0.21)$ & $-1.0(0.21)$ \\
\hline Gordon, NE & 386 & $0.8(0.10)$ & $-0.6(0.08)$ & $-0.4(0.06)$ & $-3.1(0.29)$ & $-6.2(0.21)$ & $-2.8(0.22)$ \\
\hline O'Neill, NE & 318 & $0.8(0.10)$ & $-0.5(0.08)$ & $-0.3(0.07)$ & $-2.8(0.32)$ & $-4.9(0.23)$ & $-0.7(0.24)$ \\
\hline Sidney, NE & 384 & $0.9(0.09)$ & $-0.6(0.07)$ & $-0.3(0.06)$ & $-0.7(0.21)$ & $-4.1(0.21)$ & $-0.3(0.16)$ \\
\hline West Point, NE & 247 & $1.3(0.14)$ & $-0.8(0.10)$ & $-0.2(0.09)$ & $-4.3(0.29)$ & $-5.7(0.25)$ & $-1.5(0.17)$ \\
\hline All 15 locations & 5,126 & $1.3(0.03)$ & $-0.5(0.03)$ & $0.1(0.020)$ & $-2.7(0.08)$ & $-4.2(0.06)$ & $0.0(0.06)$ \\
\hline
\end{tabular}

a $\mathrm{SEM}=$ standard error of the mean difference.

b Number of 24-h periods included in the analysis.

${ }^{c}$ CART/SLD = classification and regression tree/stepwise linear discriminant. 


\section{RESULTS}

The total number of days per weather station without out-of-range or missing hourly measurements ranged from 247 to 388 during the three study years. In total, 5,126 station-days were included in the data set.

The CART/SLD model with corrected wind speed (CART/SLD/Wind) estimated LWD with less error and variability than either the SkyBit Wetness model or the CART/SLD/SkyBit model (Table 2). Overall, the SkyBit Wetness model underestimated LWD by an ME of $1.4 \mathrm{~h}$ /day. ME in LWD estimation by the SkyBit Wetness model ranged among sites from -3.0 to 1.7 $\mathrm{h}$ /day. Inputting SkyBit weather data into the CART/SLD model (CART/SLD/SkyBit) resulted in mean estimation errors $>3 \times$ larger than for the SkyBit Wetness model. The CART/SLD/Wind model reduced ME and MAE in LWD estimation to $0.1 \mathrm{~h} /$ day and $4.2 \mathrm{~h} /$ day, respectively, whereas the range of ME and MAE across locations was -3.2 to $2.6 \mathrm{~h} /$ day and 3.2 to $5.0 \mathrm{~h} /$ day, respectively.

Overall, the SkyBit Wetness model overestimated LWD during the period 10:00 to 19:00 but underestimated LWD from 20:00 to 9:00 (Table 3). The magnitude of ME by the SkyBit Wetness model (Table 2), therefore, resulted from compensating errors in day and night periods. The CART/SLD/Wind model had lower estimation errors for LWD than either the SkyBit Wetness model or the CART/SLD/ SkyBit model at most sites, especially during the 20:00 to 9:00 time period.

The SkyBit Wetness model was significantly $(P<0.05)$ more accurate on nights with measured rainfall than on nights without rain at all stations (Table 4). The CART/SLD/SkyBit model consistently underestimated wetness duration, but to a greater extent during nights with rain than without rain. In contrast, estimation errors of the CART/SLD/Wind model did not differ significantly $(P<0.05)$ on rain versus nonrain nights for 10 of 15 stations, and were considerably smaller than for the other two models.

Sensitivity analysis indicated that the CART/SLD/Wind model misclassified far fewer hours (dry hours as wet or vice versa) than other models, especially when RH was high (Fig. 3). At lower wind speeds, both CART/SLD/SkyBit and CART/SLD/Wind were more accurate than the SkyBit Wetness model. At higher wind speeds, however, the CART/SLD/Wind model was more accurate that the other two models (Fig. 3).

Estimation error for RH significantly $(P$ $<0.0001)$ influenced performance of the SkyBit Wetness model and CART/SLD/ SkyBit model (Fig. 4). When RH was underestimated, it was apparent for both models that the magnitude of RH error was inversely related to the magnitude of model error. Accuracy of the CART/SLD/ Wind model, however, was not significantly correlated to $\mathrm{RH}$ estimation error $(P$ $=0.2043$ ).

\section{DISCUSSION}

This study is the first to utilize models to enhance accuracy of commercially available, site-specific estimates of LWD. These estimates have tremendous potential for accelerating the implementation of weather-based disease-warning systems (4) and have been shown to encounter considerable error in estimating LWD (5); therefore, the substantial improvement in accuracy we have documented should increase reliability of these technologies in diseasewarning systems $(5,13)$.

The much greater MAE of the SkyBit Wetness model compared with the CART/SLD/Wind model (Table 2) resulted from the fact that SkyBit underestimated wetness duration during night periods and overestimated it at other times. Gleason et al. (5), noting similar SkyBit LWD error trends in a study at 19 sites in northeastern North America, suggested that these errors might result either from inaccurate simulated inputs, especially $\mathrm{RH}$, or from inaccuracy in SkyBit's wetness-prediction algorithm.

Without a wind speed correction, the CART/SLD model using SkyBit input data substantially underestimated the duration of wet periods at all locations, presumably because wind speed from SkyBit was estimated at a height of $10 \mathrm{~m}$ rather than near the ground. Usually, wind speed aloft exceeds that near the surface because of drag force exerted by the ground. Higher wind speed at night disrupts the stable or nearly neutral conditions that favor dew formation, and facilitates evaporation of dew $(9,10,16)$. LWD underestimation by the CART/SLD/SkyBit model, therefore, probably reflects the fact that overestimated wind speed led to underestimation of surface wetness occurrence. The large errors incurred by this model emphasize the risk of applying commercially available input data to a LWD estimation model without recognizing the inherent limitations of the data set.

Truxall and Travis (19) and Gleason et al. (5) reported that SkyBit estimates were relatively accurate for air temperature. In our study, SkyBit air temperature estimates were relatively accurate $\left(R^{2}=0.93\right)$, whereas RH underestimation errors were relatively large, especially at high $\mathrm{RH}\left(R^{2}\right.$ $=0.84 ; 20)$. When input parameters differ in degree of accuracy, the choice of algorithm for a wetness estimation model may affect accuracy. Therefore, it would be important to employ a model that could limit influence of RH inaccuracy in order

Table 4. Mean error (ME) of model-estimated wetness duration (h/period) from 20:00 to 9:00, with and without measured rainfall

\begin{tabular}{|c|c|c|c|c|c|c|c|c|}
\hline \multirow[b]{3}{*}{ City } & \multirow[b]{3}{*}{$n_{1}^{b}$} & \multirow[b]{3}{*}{$n_{2}{ }^{b}$} & \multicolumn{6}{|c|}{ ME $(\text { SEM })^{a}$} \\
\hline & & & \multicolumn{2}{|c|}{ SkyBit Wetness } & \multicolumn{2}{|c|}{ CART/SLD/SkyBit } & \multicolumn{2}{|c|}{ CART/SLD/Wind } \\
\hline & & & No rain & Rain & No rain & Rain & No rain & Rain \\
\hline Ames, IA & 218 & 60 & $-4.4(0.35)$ & $1.6(0.57)^{* *}$ & $-4.1(0.30)$ & $-6.2(0.48)^{* *}$ & $-0.1(0.28)$ & $0.3(0.49)$ \\
\hline Lewis, IA & 324 & 56 & $-0.7(0.24)$ & $2.4(0.55)^{* *}$ & $-1.7(0.19)$ & $-4.9(0.43)^{* *}$ & $2.3(0.19)$ & $1.6(0.39)$ \\
\hline Nashua, IA & 212 & 64 & $-2.6(0.33)$ & $1.8(0.49)^{* *}$ & $-2.4(0.25)$ & $-5.4(0.45)^{* *}$ & $1.7(0.22)$ & $1.6(0.32)$ \\
\hline Sutherland, IA & 321 & 56 & $-3.6(0.29)$ & $0.6(0.62)^{* *}$ & $-4.5(0.22)$ & $-7.4(0.50)^{* *}$ & $0.4(0.22)$ & $-0.4(0.50)$ \\
\hline Crawfordsville, IA & 320 & 40 & $-4.2(0.30)$ & $2.6(0.73)^{* *}$ & $-4.1(0.23)$ & $-4.9(0.65)$ & $0.2(0.21)$ & $2.3(0.64)^{* *}$ \\
\hline Belleville, IL & 227 & 40 & $-4.1(0.38)$ & $1.7(0.74)^{* *}$ & $-2.2(0.27)$ & $-2.7(0.57)$ & $0.7(0.29)$ & $2.8(0.42)^{* *}$ \\
\hline Bondville, IL & 269 & 58 & $-4.7(0.34)$ & $0.3(0.62)^{* *}$ & $-4.9(0.25)$ & $-6.1(0.54)$ & $-0.5(0.25)$ & $1.1(0.54)^{*}$ \\
\hline Dixon Springs, IL & 280 & 45 & $-3.9(0.25)$ & $0.5(0.47)^{* *}$ & $-2.1(0.21)$ & $-4.6(0.55)^{* *}$ & $0.8(0.18)$ & $1.0(0.36)$ \\
\hline Monmouth, IL & 277 & 45 & $-3.5(0.35)$ & $2.0(0.64)^{* *}$ & $-3.6(0.26)$ & $-5.6(0.75)^{* *}$ & $0.3(0.27)$ & $1.5(0.60)$ \\
\hline St. Charles, IL & 276 & 43 & $-4.0(0.34)$ & $2.0(0.46)^{* *}$ & $-4.6(0.22)$ & $-5.6(0.50)$ & $-1.0(0.26)$ & $0.9(0.37)^{* *}$ \\
\hline Red Cloud, NE & 305 & 83 & $-4.2(0.29)$ & $1.2(0.51)^{* *}$ & $-5.1(0.24)$ & $-7.1(0.40)^{* *}$ & $-1.1(0.25)$ & $-0.4(0.36)$ \\
\hline Gordon, NE & 306 & 80 & $-3.9(0.33)$ & $0.4(0.40)^{* *}$ & $-5.7(0.24)$ & $-7.9(0.38)^{* *}$ & $-2.8(0.25)$ & $-2.5(0.43)$ \\
\hline O'Neill, NE & 250 & 64 & $-3.6(0.35)$ & $0.2(0.71)^{* *}$ & $-4.7(0.26)$ & $-6.0(0.53)^{*}$ & $-0.7(0.27)$ & $-0.8(0.55)$ \\
\hline Sidney, NE & 308 & 76 & $-1.2(0.23)$ & $1.4(0.46)^{* *}$ & $-3.1(0.20)$ & $-8.2(0.42)^{* *}$ & $-0.1(0.18)$ & $-1.4(0.34)^{* *}$ \\
\hline West Point, NE & 198 & 49 & $-5.1(0.31)$ & $-1.1(0.52)^{* *}$ & $-5.5(0.27)$ & $-6.7(0.53)^{*}$ & $-1.7(0.19)$ & $-1.0(0.28)$ \\
\hline All 15 locations & 4,091 & 859 & $-3.5(0.08)$ & $0.9(0.15)$ & $-3.9(0.07)$ & $-6.1(0.14)$ & $-0.1(0.06)$ & $0.1(0.13)$ \\
\hline
\end{tabular}

a SEM $=$ standard error of the mean difference; CART/SLD = classification and regression tree/stepwise linear discriminant. * and $* *$ indicate significant $(P<0.05)$ and highly significant $(P<0.01)$ differences, respectively, between ME for periods with and without measured rainfall.

b $n_{1}$ is the number of periods from 20:00 to 9:00 without measured rainfall and $n_{2}$ is the number of periods from 20:00 to 9:00 with measured rainfall. 
to estimate LWD as accurately as possible with SkyBit input data. With energy balance or physical models in which all inputs are considered simultaneously, inaccuracy in a single input parameter may greatly influence model performance because errors in input values can be preserved or even amplified (11). For example, Magarey (13), using a physical model, attributed unacceptably large errors in wetness-duration estimation to inaccuracy in site-specific $\mathrm{RH}$ inputs.

The hierarchical nature of the CART procedure, however, may improve accuracy of LWD estimation using SkyBit input data. In the CART procedure, each input variable contributes to the wetness prediction process in a sequential manner, so that the impact of inaccuracy of an individual variable on model performance can be reduced. Since the CART procedure can isolate severely underestimated RH at two steps, DPD and RH (Fig. 2), the influence of these underestimated values on model performance was greatly reduced. At $\mathrm{RH}>$ $80 \%$, for example, the CART/SLD/Wind model produced a much lower error than the SkyBit Wetness model.

Applying a wind speed correction also improved performance of the CART/SLD/ Wind model. For example, the wind speed correction clearly contributed to enhanced accuracy of wetness estimation at the high (>80\%) RH under which wetness was typically measured. The SkyBit Wetness model also has a wind speed correction process to $1.0 \mathrm{~m}$ (J. Russo, SkyBit, Inc., personal communication). The fact that performance of the SkyBit Wetness model was no better than that of the CART/SLD/SkyBit model below the seventy-fifth percentile of wind speed, however, suggests that the wind speed correction of the former was not sufficient to improve model performance over most of the range of conditions we observed.

Accordingly, both the hierarchical structure of the CART procedure and the wind speed correction allowed the CART/SLD/ Wind model to estimate LWD with reasonable accuracy despite relatively inaccurate SkyBit estimation of RH. Correlation between the magnitude of estimate error for $\mathrm{RH}$ and accuracy was not significant, implying that the CART/SLD model approach was robust to the influence of erroneous RH values. A potential additional advantage of the CART/SLD model is that, unlike energy balance models, the CART/SLD model does not rely on net
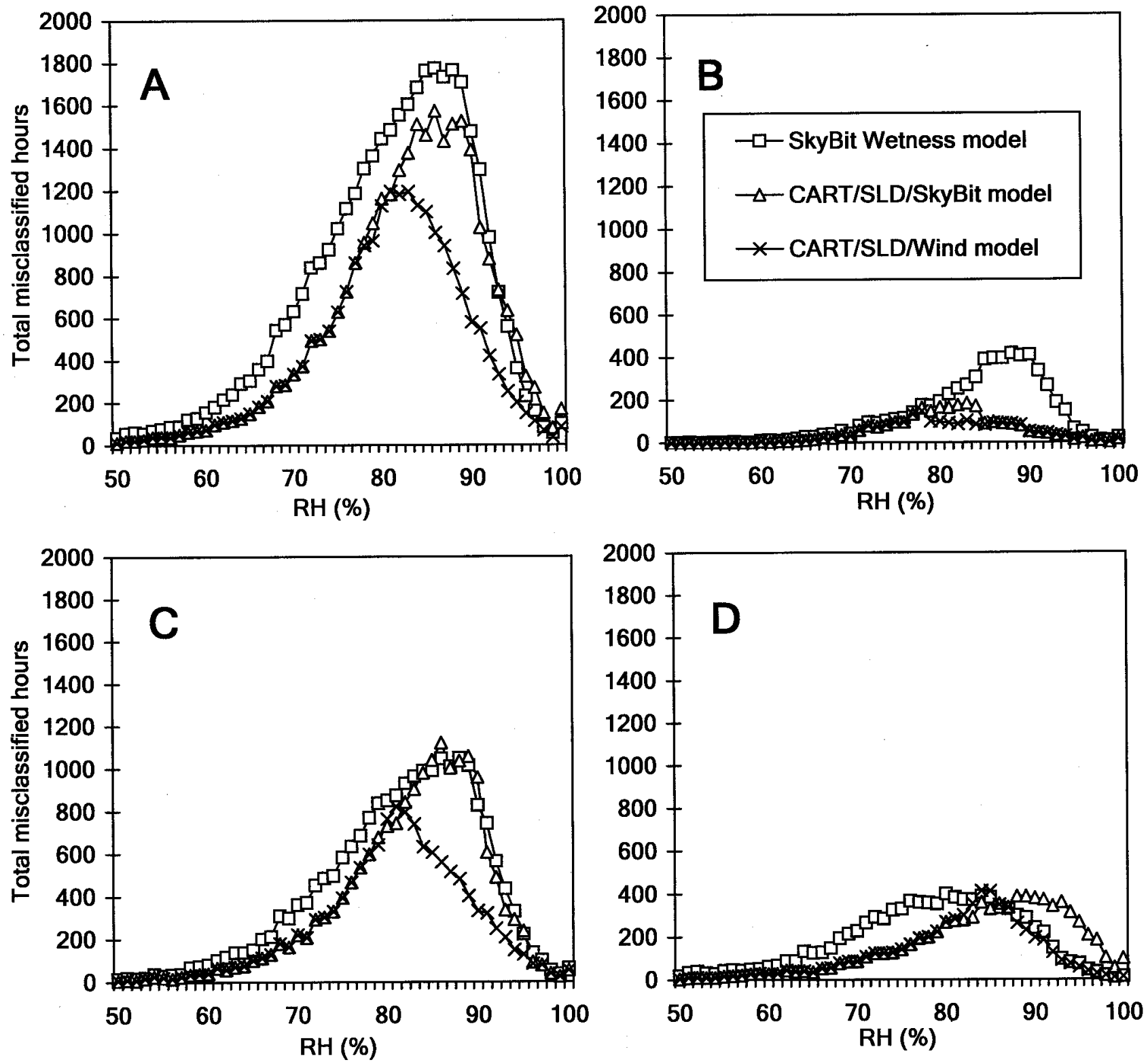

Fig. 3. Model response to relative humidity (RH) at different ranges of wind speed: $\mathbf{A}$, full range $(0 \leq x \leq 13.0 \mathrm{~m} / \mathrm{s})$; $\mathbf{B}$, below the twenty-fifth percentile $(<1.3 \mathrm{~m} / \mathrm{s})$; C, between the twenty-fifth and seventy-fifth percentile $(1.3 \mathrm{~m} / \mathrm{s} \leq x \leq 3.6 \mathrm{~m} / \mathrm{s})$; D, above the seventy-fifth percentile $(>3.6 \mathrm{~m} / \mathrm{s})$, where $x=$ wind speed. Lines represent total number of hours in which wetness status was misclassified (e.g., measured dry hours were misclassified as wet or vice versa) at indicated $\mathrm{RH}$. 


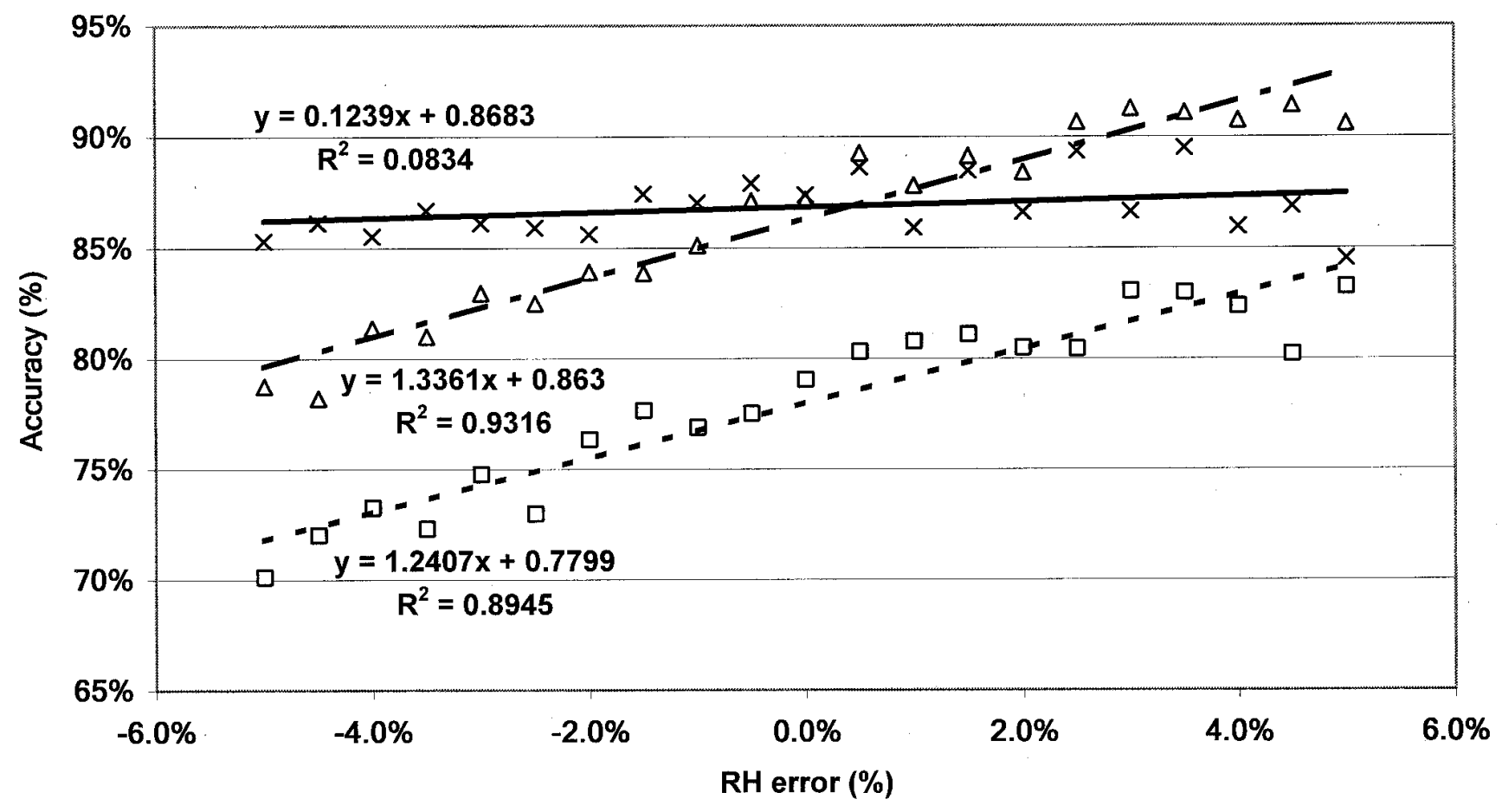

\section{口 SkyBit Wetness}

- - - Linear (SkyBit Wetness) $\triangle$ CART/SLD/SkyBit

- Linear (CART/SLD/SkyBit)

\section{$\times$ CART/SLD/Wind}

Linear (CART/SLD/Wind)

Fig. 4. Relationship between error of SkyBit relative humidity (RH) estimate and accuracy of wetness event prediction. Accuracy is defined here as the proportion of hours in which occurrence or absence of wetness was estimated correctly. RH error and respective accuracy (\%) of the SkyBit Wetness model and the classification and regression tree/stepwise linear discriminant (CART/SLD) SkyBit model were correlated significantly $(P<0.0001)$. For the CART/SLD/Wind model, the correlation between RH error and model error was not significant $(P=0.2043)$.

radiation data, which are difficult to estimate accurately (T. J. Gillespie, University of Guelph, Guelph, Ontario, Canada, personal communication).

Advantages of using remotely estimated weather data to estimate LWD include not only cost effectiveness, by saving the expense of installing, downloading, and maintaining an automated weather station at each site, but also extension of potential service areas. Francl and Panigrahi (2) suggested that a wetness estimation model should demonstrate both transferability to different environments and repeatability in similar environments before it is deployed in the field. Based on our results at 15 sites in the Midwest over 3 years, the CART/SLD/Wind model using SkyBit input data may be a suitable choice for LWD estimation where measured weather data are not available in the region. Results for the CART/SLD/Wind models with SkyBit input data suggest that reasonably accurate estimations of LWD can be achieved even when locally measured weather data are unavailable.

The CART/SLD model with wind speed correction has the potential to accurately identify periods of environmental wetness in the midwestern United States and, therefore, merits further attention as a tool to enhance accuracy and user acceptability of commercially available, remote estimates of weather parameters.

\section{ACKNOWLEDGMENTS}

We thank S. Wegulo and R. W. Arritt, Iowa State University, and T. J. Gillespie, University of Guelph, for reviewing this manuscript; and R. Scott and S. Hollinger of the Midwest Climate Center, (Champaign, IL), S. Hanneman, K. Blauvelt, and K. Hubbard of the High Plains Climate Center (Lincoln, NE), and K. Berns, Iowa State University, for their cooperation in gathering and compiling weather data. Supported by grant no. 98-34103-6118 of the USDA North Central Integrated Pest Management Grants Program.

\section{LITERATURE CITED}

1. Davis, D. R., and Hughes, J. E. 1970. A new approach to recording the wetting parameter by the use of electrical resistance sensors. Plant. Dis. Rep. 54:474-479.

2. Francl, L. J., and Panigrahi, S. 1997. Artificial neural network models of wheat leaf wetness. Agric. For. Meteorol. 88:57-65.

3. Gillespie, T. J., and Kidd, G. E. 1978. Sensing duration of leaf moisture retention using electrical impedance grids. Can. J. Plant. Sci. 58:179-187.

4. Gleason, M. L. 2000. Disease-warning systems. Pages 367-370 in: Encyclopedia of Plant Pathology. Vol I. John Wiley \& Sons, New York.

5. Gleason, M. L., Parker, S. K., Pitblado, R. E., Latin, R. X., Speranzini, D., Hazzard, R. V., Maletta, M. J., Cowgill, W. P., Jr., and Biederstedt, D. L. 1997. Validation of a commercial system for remote estimation of wetness duration. Plant Dis. 81:825-829.

6. Gleason, M. L, Taylor, S. E., Loughin, T. M., and Koehler, K. J. 1994. Development of validation of an empirical model to estimate the duration of dew periods. Plant Dis. 78:10111016.

7. Haas, J. H., and Rotem., J. 1976. Pseudomonas lachrymans inoculum on infected cucumber leaves subjected to dew-and rain-type wetting. Phytopathology 66:1219-1223.

8. Huber, L., and Gillespie, T. J. 1992. Modeling leaf wetness in relation to plant disease epidemiology. Annu. Rev. Phytopathol. 30:553-77.

9. Jacobs, A. F. G, van Boxel, J. H., and Shaw, R. H. 1992. The dependence of canopy layer turbulence on within-canopy thermal stratification. Agric. For. Meteorol. 58:247-256.

10. Jacobs, A. F. G, van Boxel, J. H., and Shaw, R. H. 1992. Horizontal and vertical distribution of air temperature in a vegetation canopy. Neth. J. Agric. Sci. 40:359-372.

11. Kincaid, D., and Cheney, W. 1996. Numerical Analysis: Mathematics of Scientific Computing. 2nd ed. Brooks/Cole, Pacific Grove, CA.

12. Lau, Y. F., Gleason, M. L., Zriba, N., Taylor, S. E., and Hinz, P. N. 2000. Effects of coating, deployment angle, and compass orientation on performance of electronic wetness sensors during dew periods. Plant Dis. 84:192-197.

13. Magarey, R. D. 1999. A theoretical standard for estimation of surface wetness duration in grape. Ph.D. dissertation. Cornell University, Ithaca, NY.

14. Pedro, M. J., and Gillespie, T. J. 1982. Estimating dew duration. II. Utilizing standard weather station data. Agric. Meteorol. 25:297310.

15. Potratz, K. J., Gleason, M. L., Hockmuth, M. 
L., Parker, S. K., and Pearston, G. A. 1994. Testing the accuracy and precision of wetness sensors in a tomato field and on turfgrass. J. Iowa Acad. Sci. 101(2):56-60.

16. Rosenberg, N. J., Blad, B. L., and Verma, S. B. 1983. Microclimate: The Biological Environment. 2nd ed. John Wiley \& Sons, New York.

17. Schuh, W. 1993. Influence of interrupted dew periods, relative humidity, and light on dis- ease severity and latent infections caused by Cerospora kikuchii on soybean. Phytopathology 83:109-113.

18. Stull, R. B. 2000. Meteorology for Scientists and Engineers. 2nd ed. Brooks/Cole, Pacific Grove, CA.

19. Truxall, D. L., and Travis, J. W. 1994. Analysis of the accuracy of predicted weather data on the impact on apple disease management. (Abstr.) Phytopathology 84:1125.
20. Wegulo, S. N. Gleason, M. L. Kim, K. S Taylor, S. E., and Koehler, K. J. 2001. Impact of accuracy and precision of site-specific estimation of weather data in the midwestern USA on simulated performance of disease-warning systems. (Abstr.) Phytopathology 91:S94.

21. Wilks, D. S., and Shen, K. W. 1991. Threshold relative humidity duration forecasts for plant disease prediction. J. Appl. Meteorol. 30:463-470. 